\title{
ARTICLE cDNA-AFLP analysis reveals differential gene expression in incompatible interaction between infected non-heading Chinese cabbage and Hyaloperonospora parasitica
}

\author{
Dong Xiao ${ }^{1,2}$, Shi-Tuo Liu ${ }^{1,2}$, Yan-Ping Wei ${ }^{1,2}$, Dao-Yun Zhou ${ }^{1,2}$, Xi-Lin Hou ${ }^{1,2}$, Ying $\mathrm{Li}^{1,2}$ and Chun-Mei Hu ${ }^{1,2}$
}

Non-heading Chinese cabbage (Brassica rapa ssp. chinensis) is one of the main green leafy vegetables in the world, especially in China, with significant economic value. Hyaloperonospora parasitica is a fungal pathogen responsible for causing downy mildew disease in Chinese cabbage, which greatly affects its production. The objective of this study was to identify transcriptionally regulated genes during incompatible interactions between non-heading Chinese cabbage and $H$. parasitica using complementary DNA-amplified fragment length polymorphism (cDNA-AFLP). We obtained 129 reliable differential transcript-derived fragments (TDFs) in a resistant line 'Suzhou Qing'. Among them, 121 upregulated TDFs displayed an expression peak at 24-48 $\mathrm{h}$ post inoculation (h.p.i.). Fifteen genes were further selected for validation of cDNA-AFLP expression patterns using quantitative reverse transcription PCR. Results confirmed the altered expression patterns of 13 genes (86.7\%) revealed by the cDNA-AFLP. We identified four TDFs related to fungal resistance among the 15 TDFs. Furthermore, comparative analysis of four TDFs between resistant line 'Suzhou Qing' and susceptible line 'Aijiao Huang' showed that transcript levels of TDF14 (BCLIK1_A01) peaked at 48 h.p.i. and 25.1-fold increased in the resistant line compared with the susceptible line. Similarly, transcript levels of the other three genes, TDF42 (BCCAT3_A07), TDF75 (BCAAE3_A06) and TDF88 (BCAMT2_A05) peaked at 24, 48 and 24 h.p.i. with 25.1-, 100- and 15.8-fold increases, respectively. The results suggested that the resistance genes tended to transcribe at higher levels in the resistance line than in the susceptible line, which may provide resistance against pathogen infections. The present study might facilitate elucidating the molecular basis of the infection process and identifying candidate genes for resistance improvement of susceptible cultivars.

Horticulture Research (2016) 3, 16034; doi:10.1038/hortres.2016.34; Published online 27 July 2016

\section{INTRODUCTION}

It is well known that plant-pathogen interactions activate a subset of pathogen genes so-called systemic acquired resistance to protect themselves. ${ }^{1-3}$ This interaction process is diverse and complicated because plant pathogens have evolved by developing various strategies to infect their hosts. Specific pathogen may trigger defense systems that are essential for pathogenicity. Next, molecular responses are up- or downregulations by numerous specific resistant genes. During the development of interaction, the recognition of specific host genes determines whether the interaction will be successful.

Downy mildew is an important fungal disease of Brassica specie that is caused by the obligatory biotrophic oomycete Hyaloperonospora parasitica (formerly Peronospora parasitica (Pers. Ex Fr.)), and infects most members of the Brassica family. ${ }^{4}$ It can be fatal to seedling growth in the nurseries and reduce the productivity and quality of adult plants in the field. ${ }^{4}$ Leaves become yellow after infection and then scorch. When downy mildew became epidemic, it can cause damage to $>90 \%$ of the crop. The disease is more severe in spring and autumn seasons than in other seasons. Currently, the downy mildew disease is controlled by application of fungicides. ${ }^{5}$ However, chemical control is often difficult and ineffective. It has been proved that the most efficient way to manage plant diseases is to develop a host resistance in new cultivars. ${ }^{5}$
Therefore, to identify the host resistant genes is a crucial need for obtaining reliable resistant genotypes to assist plant breeding. Previous studies have shown some differentially expressed genes during infection process using various methods. ${ }^{6,7}$ The differential display-based strategy has been used to reveal genes related to downy mildew infection in $B$. oleracea seedlings. ${ }^{6}$ Suppression subtractive hybridization technology has been employed and revealed 37 high-quality Expressed Sequence Tags (ESTs), of which functions are known in energy metabolism, transcriptional regulation, signal transduction and defense reaction. ${ }^{7}$ However, most molecular components of the signal transduction pathway involved in gene regulation remain to be identified.

Furthermore, there is no report on pathogen virulence genes matching the resistance genes of non-heading Chinese cabbage (Brassica rapa ssp. chinensis), and their inheritance remains uncertain. In this prospect, it is important to elucidate the molecular mechanisms or gene expression profile and to identify an inventory of candidate genes during the non-heading Chinese cabbage- $H$. parasitica interaction.

Screening for differentially expressed genes is a direct approach to reveal the molecular basis of a biological system. The complementary DNA-amplified fragment length polymorphism (cDNA-AFLP) method has been successfully used for the identification of genes involved in various plant-pathogen 
systems. ${ }^{8,9}$ In comparison with microarray technique and RNA sequencing, cDNA-AFLP costs less and does not require sequence information. When compared with subtractive hybridization, CDNA-AFLP is highly reproducible. ${ }^{8}$

The objectives of this study were to apply the cDNA-AFLP technique to the pathogenic interaction between non-heading Chinese cabbage and downy mildew. We identified a set of genes that were regulated during the incompatible interaction between the host and pathogen, and validated the expression patterns for the regulated genes.

\section{MATERIALS AND METHODS}

Plant material, inoculums and pathogen infection

Two non-heading Chinese cabbage inbred lines, 'Suzhou Qing' (resistant to $H$. parasitica) and 'Aijiao Huang' (susceptible to $H$. parasitica) from our lab, were used in this study. The transcript-derived fragments (TDFs) were obtained from interaction between 'Suzhou Qing' and downy mildew (H. parasitica). 'Aijiao Huang' was used for the comparison of expression patterns of the four genes related with fungal resistance between resistant and susceptible line.

Plants were grown in plastic nurseries (inner size: $45 \times 45 \mathrm{~mm}$; height: $57 \mathrm{~mm}$ ) and transferred to a growth chamber under $25^{\circ} \mathrm{C}$ day/ $20^{\circ} \mathrm{C}$ night temperature with $85 \pm 5 \%$ relative humidity and a 12-h light/12-h dark after germination for $36 \mathrm{~h}$ under dark. H. parasitica was isolated from leaves of susceptible line 'Aijiao Huang' in the Jiangpu Farm of Nanjing Agricultural University, China. ${ }^{10}$ Conidial suspensions were adjusted to $1 \times 10^{5}$ spores per $\mathrm{mL}$ and Tween-20 was added as a surfactant to a final concentration of $0.1 \%$. One hundred of 3-week-old seedlings (with four true-leaves) were sprayed with $50 \mathrm{~mL}$ pathogen suspension and distilled water (as control), respectively. After inoculation, the seedlings were covered with plastic film separately and transferred to a growth chamber under $20^{\circ} \mathrm{C}, 100 \%$ relative humidity in the dark for the first $24 \mathrm{~h}$ to promote sporulation, then moved back to the initiatory conditions. Both control and treated third leaf of five plants were harvested and pooled at 0, 24, 48 and $72 \mathrm{~h}$ post inoculation (h.p.i.), immediately frozen in liquid nitrogen and stored at $-70{ }^{\circ} \mathrm{C}$ until use.

\section{RNA isolation and CDNA-AFLP analysis}

Total RNAs were extracted using the RNAeasy Plant Mini kit (Qiagen; https://www.qiagen.com/cn/shop/sample-technologies/rna/rna-preparation/ rneasy-mini-kit\#orderinginformation) and synthesis of the first strand of CDNA is made using the M-MLV reverse transcriptase (Takara Shuzo Co., Ltd, Japan) according to the manufacturer's protocol. To synthesize the second strand, the following components were added to the first-strand solution. A volume of $30 \mu \mathrm{L}, 5 \times 2$ nd strand synthesis buffer, $3 \mu \mathrm{L}$ dNTP mixture, $89 \mu \mathrm{L}$ RNase-free $\mathrm{H}_{2} \mathrm{O}, 2 \mu \mathrm{L}$ Escherichia coli DNA polymerase I, $2 \mu \mathrm{L}$ E. coli RNase $\mathrm{H} / \mathrm{E}$. coli DNA ligase mixture and $4 \mu \mathrm{L} \mathrm{T}_{4}$ DNA polymerase in a final volume of $150 \mu \mathrm{L}$. The components were gently mixed and incubated at $16^{\circ} \mathrm{C}$ for $2 \mathrm{~h}$. Double-stranded CDNA was purified using the DNA Fragment Purification Kit Ver.2.0 (Takara).

Second-strand CDNA was digested by two restriction enzymes Tag I (restriction site TCGA) and Ase I (restriction site ATTAAT; Takara), and then ligated to Tag $I$ and Ase I double-strand adaptors. The AFLP adaptor primers 5'-GACGATGAGTCCTGAC-3', 5'-CGGTCAGGACTCAT-3' (Taq l-adaptor primers) and 5'-GCGTAGACTGCGTACC-3', 5'-TAGGTACGCAGTC-3' (Ase 1 -adaptor primers) were ligated onto the restriction fragments: Taq I pre-amplification primer, 5'-GACGATGAGTCCTGACCGA-3'; Ase I pre-amplification primer, 5-CTCGTAGACTGCGTACCTAAT-3'; Taq / selective amplification primer, 5'-GATGAGTCCAGACCGA+NN-3'; Ase I selective amplification primer, 5'-GACTGCGTACCTAAT+NN-3' (indicated by $\mathrm{N}$, representing an A, C, G or T). The initial small-scale screen using 96 AFLP primer combinations were done using six Taq I forward selective amplification primers (extension CG, CA, CT, CC, GA or GT) in combination with 16 Ase I reverse selective amplification primers (extension NN), respectively. Pre-amplification PCR was carried out with one-tenth volume of the restriction/ligation mix, the pre-amplification PCR was carried out as follows: $94{ }^{\circ} \mathrm{C}, 3 \mathrm{~min} ; 94^{\circ} \mathrm{C}, 30 \mathrm{~s}, 55^{\circ} \mathrm{C}, 30 \mathrm{~s}, 72{ }^{\circ} \mathrm{C}, 60 \mathrm{~s}, 25$ cycles; and $72{ }^{\circ} \mathrm{C}$, $5 \mathrm{~min}$. The products of pre-amplification was diluted 10 -fold, and the selective amplification PCR was carried out as follows: $94{ }^{\circ} \mathrm{C}, 30 \mathrm{~s} ; 94^{\circ} \mathrm{C}$, $30 \mathrm{~s}, 65^{\circ} \mathrm{C}, 30 \mathrm{~s}\left(-0.7^{\circ} \mathrm{C}\right.$ per cycle), $72{ }^{\circ} \mathrm{C}, 60 \mathrm{~s}, 12$ cycles; $94^{\circ} \mathrm{C}, 30 \mathrm{~s}, 56^{\circ} \mathrm{C}$, $30 \mathrm{~s}, 72^{\circ} \mathrm{C}, 60 \mathrm{~s}, 24$ cycles; and $72^{\circ} \mathrm{C}, 5 \mathrm{~min}$.

Selective amplification products were separated on a $6 \%$ polyacrylamide gels running at $60 \mathrm{~W}$ for $2 \mathrm{~h}$ and visualized by silver staining. Differential bands were excised from the polyacrylamide gel electrophoresis gels based on the alignment between films and markers on the gels, and incubated in $30 \mu \mathrm{L}$ of water and then at $95^{\circ} \mathrm{C}$ for $30 \mathrm{~min}$. The TDFs were then re-amplified by PCR using same primers under the similar conditions. The amplified fragments were retrieved from a $1 \%$ agarose gel with the Sephaglas BandPrep kit (Amersham Pharmacia Biotech.), cloned into pGEM-T Easy vector (Takara) according to the manufacturer's protocol and sequenced by Invitrogen Company (Shanghai BioWisdom Technology Co, Ltd; Shanghai, China. http://en.cellfood.com.cn/culture. aspx), and sequence information was BLASTed in the Brassica database (http://brassicadb.org/brad/).

\section{Quantitative real-time PCR}

The single-strand cDNA of resistant line 'Suzhou Qing' and susceptible line 'Aijiao Huang' were diluted to $30 \mathrm{ng} \mathrm{LL}^{-1}$, and were used for quantitative reverse transcription PCR (qRT-PCR) analyses. Primers were designed by the Primers 3 (http://frodo.wi.mit.edu/primer3/) based on the interested CDNA sequence. The qRT-PCR reaction mixtures contained $12.5 \mu \mathrm{L}$, $2 \times$ SYBR Green PCR MasterMix (Applied Biosystems; http://www.bio-rad. $\mathrm{com} /$ ), $10 \mathrm{pm}$ of each primer, $2 \mu \mathrm{L}$ template and sterile distilled water to total volume of $25 \mu \mathrm{L}$, as well as also performed on CFX96 Real-Time System (C1000 Thermal Cycler, Bio-Rad, CA, USA). Thermal conditions were 2 min of denaturation at $95^{\circ} \mathrm{C}$, followed by 45 cycles of $95^{\circ} \mathrm{C}$ for $10 \mathrm{~s}$, annealing at $55^{\circ} \mathrm{C}$ for $20 \mathrm{~s}$, and extension at $72{ }^{\circ} \mathrm{C}$ for $20 \mathrm{~s}$ and $72{ }^{\circ} \mathrm{C}$ for $5 \mathrm{~min}$. Three technical replicates were analysed for each biological replicate. All the cycle threshold $(\mathrm{Ct})$ values from one gene were determined at the same threshold fluorescence value of 0.2 using the $\Delta \Delta \mathrm{Ct}$ method. ${ }^{11}$ The primers of gene-specific and housekeeping gene were listed in Table 1.

Statistical analysis was performed using Student's $t$-test.

\section{RESULTS}

Isolation of differentially expressed genes

To determine the early events involved during the non-heading Chinese cabbage- $H$. parasitica interactions, four gene pools were constructed from resistant inbred line 'Suzhou Qing' at 0, 24, 48 and 72 h.p.i., respectively. TDFs displayed by cDNA-AFLP analysis ranged in size from 100 to $800 \mathrm{bp}$, depending on 96 selective primer combinations and time points. Figure 1 showed an example of the expression patterns of the genes revealed using CDNA-AFLP. A total of 180 fragments were obtained with the 96 primer pairs. After excluding repeat and error sequences, 129 TDFs were obtained. Of the 129 TDFs, 121 were upregulated and 8 downregulated. Of the 121 TDFs upregulated, 35 (28.9\%), 31 (25.6\%) and 4 (3.3\%) TDFs were induced strongly at 24, 48 and 72 h.p.i., respectively; 12 (9.9\%) and 2 (1.7\%) TDFs were induced at 24 and 48 h.p.i., and 48 and 72 h.p.i., respectively. These results showed that non-heading Chinese cabbage has mainly accumulated expression at 24-48 h.p.i. and that gene expression patterns were different and complex after $H$. parasitica infection.

\section{Gene sequence analysis}

By BLASTn search on Brassica database, 129 TDFs were successfully annotated (Table 2). One hundred and sixteen TDFs (90\%) of the 129 TDFs can be divided into six functional categories, including defense (D), signal transduction (ST), energy metabolism $(E M)$, regulation $(R)$, protein-protein interaction $(P I)$, others $(O)$ and unknown (Un; Figure 2). Forty-one TDFs (31.8\%) of the annotated sequences were associated with defense. Among them, four TDFs were associated with the pathogenesis-related protein, include $\beta$ 1,3-glucanase (TDF3), hapless 8 (TDF8), pathogenesis-related protein (TDF28) and thaumatin-like protein (TDF16), and others had hypersensitive-induced response protein (TDF22), mannose-binding lectin superfamily protein (TDF23), respiratory burst oxidase protein (TDF13) and so on. Thirty-two TDFs (24.8\%) were involved in signal transduction, for example, a member of the BEL family of homeodomain proteins (TDF49), catalase (TDF42), calcium ion binding (TDF48) and so on. Followed that, 19 TDFs (14.7\%) mainly 


\begin{tabular}{|c|c|c|c|c|}
\hline D & TDF1 & BCASN1_A06 & TTCCTTCTACGCCTTATG & GAATCAAGACCACCAGAT \\
\hline D & TDF11 & BCTPI_A04 & CTCAAGTTCCTTCACAAGA & AGTTCACAAGCATCTCAG \\
\hline $\mathrm{D}$ & TDF14 & BCLIK1_A01 & ССTCCTCGTCTCTATCAT & ATTCCAGTTAGTCTTCTTCAA \\
\hline ST & TDF42 & BCCAT3_A07 & GTCCACACCTACACTCTA & СAACTACCTTAGCCTCTTC \\
\hline ST & TDF60 & BCKEG_A03 & GCCTTACACCGTTACATA & TTATAGCAGCAGCCATAC \\
\hline ST & TDF63 & BCSAMDC_A03 & GCCTTACACCGTTACATA & TTATAGCAGCAGCCATAC \\
\hline EM & TDF75 & BCAAE3_A06 & CCTCCGTCAACAACATTA & GGCGTCATACTTCTTCAT \\
\hline EM & TDF76 & BCLHCB1.1_A07 & GTTGAAGGTGAAGGAGAT & AATGGTCAGCAAGATTCT \\
\hline EM & TDF88 & BCAMT2_A05 & ACATTAGCGGTATTCTACA & GACACTACATTCCAGACA \\
\hline $\mathrm{R}$ & TDF91 & BCABCG36_A07 & TTGATGCTGATGAAGAGA & GGTGGCTGGATTATACTT \\
\hline
\end{tabular}

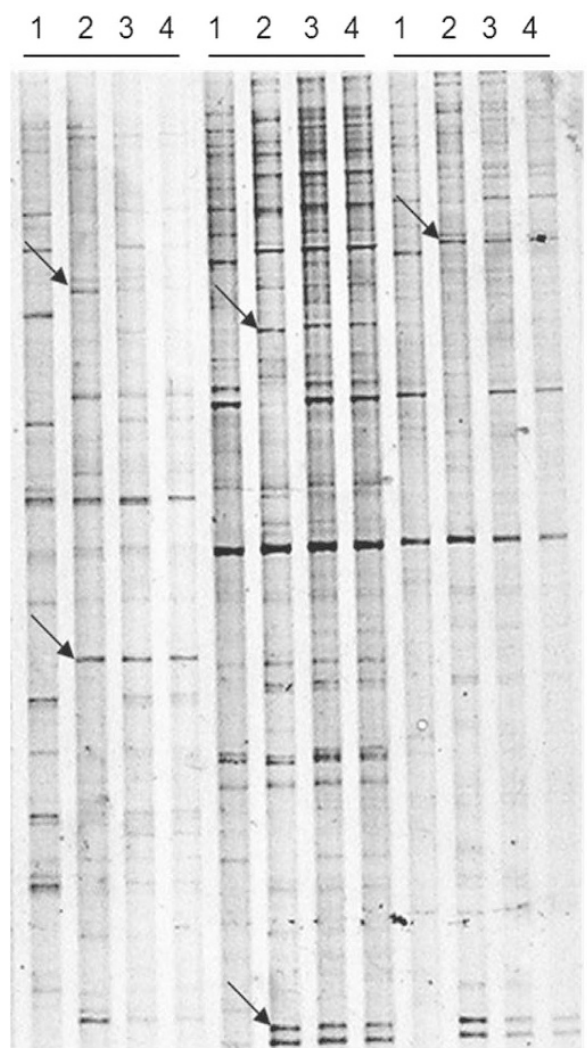

Figure 1. Expression of non-heading Chinese cabbage 'Suzhou Qing' genes in leaves inoculated with $H$. parasitica transcripts was displayed by CDNA-AFLP. An example showing that land 1, 2, 3 and 4 represents the induction time $0,24,48$ and 72 h.p.i., respectively. The size of the differential TDFs was determined by direct sequencing. Arrow: differential bands.

involved in regulation, including ATP-binding cassette G36 (TDF91), heat-shock cognate protein (TDF102) and so on. Fifteen TDFs $(11.7 \%)$ were mainly involved in energy metabolism, for example, TDF81 was predicted to involve in Arabidopsis thaliana photosynthetic electron transfer chain. Four TDFs (3.1\%) and five TDFs (3.9\%) were involved in protein-protein interaction and others metabolic pathways, respectively. These genes might function to protect cells from the fungal pathogen in non-heading Chinese cabbage. No function was assigned to 13 (10.0\%) of the TDFs as they showed no or low sequence similarities in the Brassica database search. In conclusion, gene expression patterns are more complex after infection and involved in many different metabolic pathways. It indicates that it is the common effect of these different metabolic pathways that improved the plant resistance to fungus, thereby reducing the hypersensitive response (HR) in resistance line against fungus pathogen.

Validation of expression patterns using qRT-PCR analysis

To investigate the reliability of CDNA-AFLP for detecting differentially expressed genes, qRT-PCR analysis was carried out for 15 TDFs. These TDFs were selected based on significantly different expression patterns in the time course of the CDNA-AFLP experiment and homology to genes known to have a role in defense, signal transduction, regulation and energy metabolism. Expression patterns of the 15 TDFs in non-heading Chinese cabbage leaves after infection are shown in Figure 3. The same expression pattern was found for each TDF with qRT-PCR analysis as observed in the cDNA-AFLP tests, except for TDF60 (BCKEG_A03) and TDF91 (BCABCG36_A07). As shown in Figure 3, TDFs that involved in defense (TDF1 (BCASN1_A06), TDF7 (BCCHS_A10), TDF11 (BCTPI_ A04) and TDF14 (BCLIK1_A01)) had maximum expression at 48 h.p.i., except for TDF1 that had maximum expression peaked at 24 h.p.i. TDFs involved in signal transduction included TDF42 (BCCAT3_A07), TDF49 (BCCCS_A08), TDF58 (BCNIT2_A02), TDF59 (BCRLK5_A01), TDF60 (BCKEG_A03) and TDF63 (BCSAMDC_A03)). Among of them, TDF42, TDF49 and TDF59 had similar expression patterns and peaked at 24 or 48 h.p.i. TDF58 and TDF63 had minimum expression at 72 h.p.i., suggesting that these two genes may be repressed after infection. TDFs involved in energy metabolism included TDF75 (BCAAE3_A06), TDF76 (BCLHCB1.1_ A07) and TDF88 (BCAMT2_A05). Among them, TDF75 and TDF8 8 were expressed highly at 0 h.p.i. compared with other TDFs, and showed maximum expression at 48 and 24 h.p.i., respectively. The results suggested that they may have been involved in the earlier stageinteraction between non-heading Chinese cabbage and $H$. Parasitica. TDF76 expressed very low at the 0 h.p.i. TDF91 (BCABCG36_A07) and TDF101 (BCSIG1_A08)) were related to regulation. TDF91 was slowly increased after 0 h.p.i. and maximum expression peaked at 48 h.p.i. TDF101 was strongly upregulated at 24 h.p.i. and decreased after 24 h.p.i. These results suggested that 
Table 2. Homology of obtained TDFs in the non-heading Chinese cabbage-H. parasitica interaction

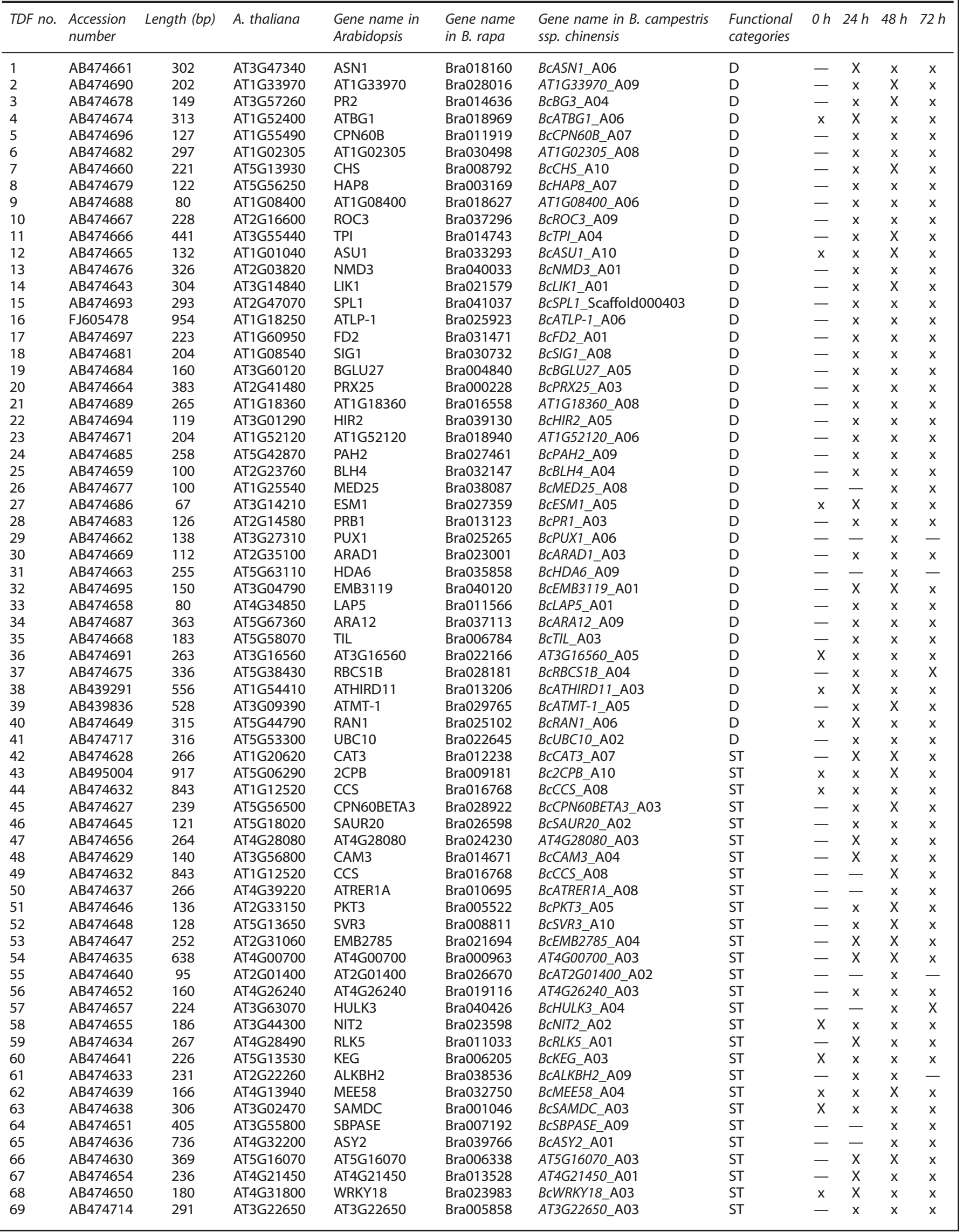




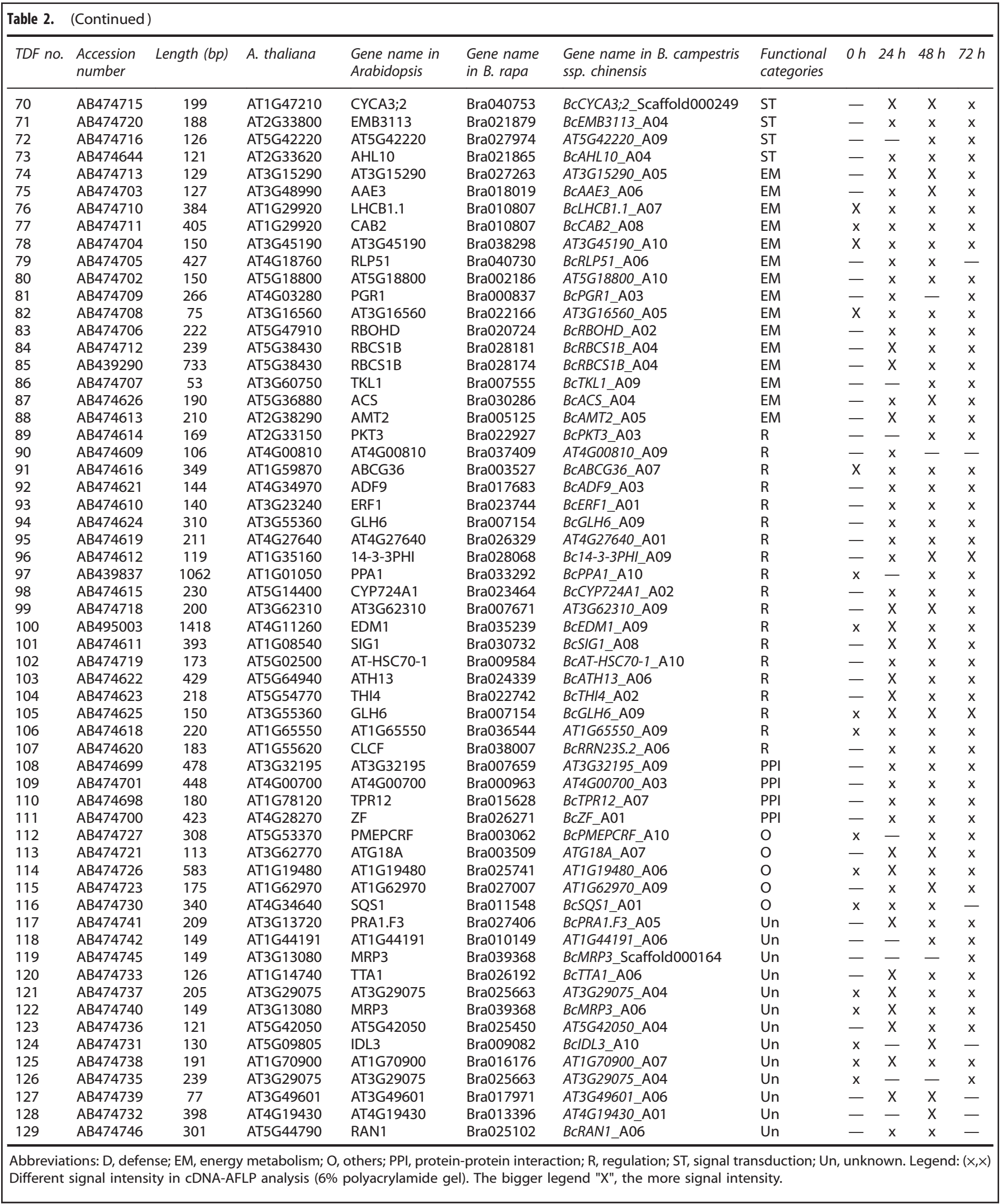

the selected TDFs with putative four categories of functions might be triggered rapidly and have an active role during the early incompatible interaction between non-heading Chinese cabbage and $H$. parasitica.
Through BLAST searching in the Arabidopsis database (http:// www.arabidopsis.org/), we found that four of the 15 TDFs were related with fungal resistance. To verify these expectations were related to fungal resistance, we performed a qRT-PCR experiment 
with a resistant and a susceptible line. Results are shown in Figure 4.

TDF14 (BCLIK1_A01) encodes LRR-RLK protein, is involved in regulation of innate immune response, and have a role against pathogens according to the homologous alignment in the

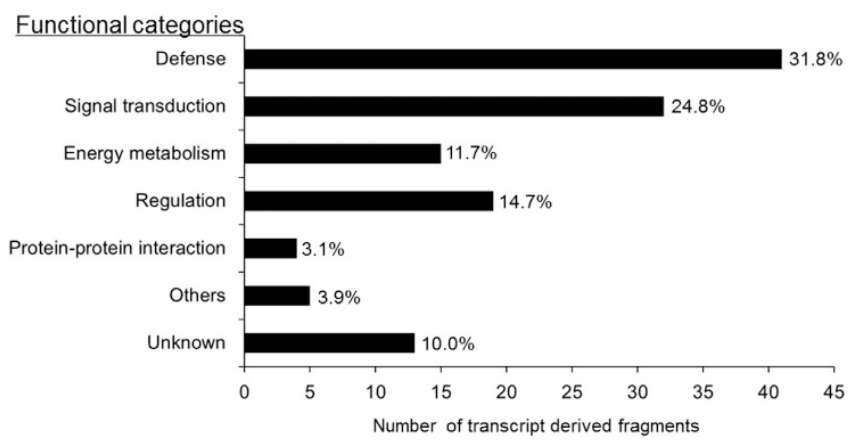

Figure 2. Classification of differentially accumulated TDFs after inoculation of $H$. parasitica. A total of 129 TDFs were classified based on the Chinese cabbage database.
Brassica database. As shown in Figure 4, gene expression of TDF14 was increased slowly after inoculation and expression peaked at 48 h.p.i. Although both have the same expression trends, the expression of TDF14 in resistant line 'Suzhou Qing' is higher than that in susceptible line 'Aijiao Huang', especially in 48 h.p.i.

TDF42 (BCCAT3_A07) encodes catalase and is involved in the regulation of defense. Catalase is one of the key enzymes in vivo anti-oxidative defense systems, which has a special role in removing the hydrogen peroxide to avoid the body to produce oxidative stress in the process. The expression patterns of TDF42 in two lines were similar to that of TDF14. TDF75 (BCAAE3_A06) encodes an oxalyl-COA synthetase and involved in defense response to fungus. The gene expression in resistant line 'Suzhou Qing' was much higher than that in susceptible line 'Aijiao Huang', with fold change reaching to 100 times at 48 h.p.i.

TDF88 (BCAMT2_A05) encodes a high-affinity ammonium transporter and involved in ammonium transmembrane transport and defense response to fungus. The expression of TDF88 was almost the same in 0 h.p.i. in two lines, strongly induced subsequently and maximum expression both peaked at 24 h.p.i. But gene expression of TDF88 in resistant line 'Suzhou Qing' was always higher than that in susceptible line 'Aijiao Huang'. The
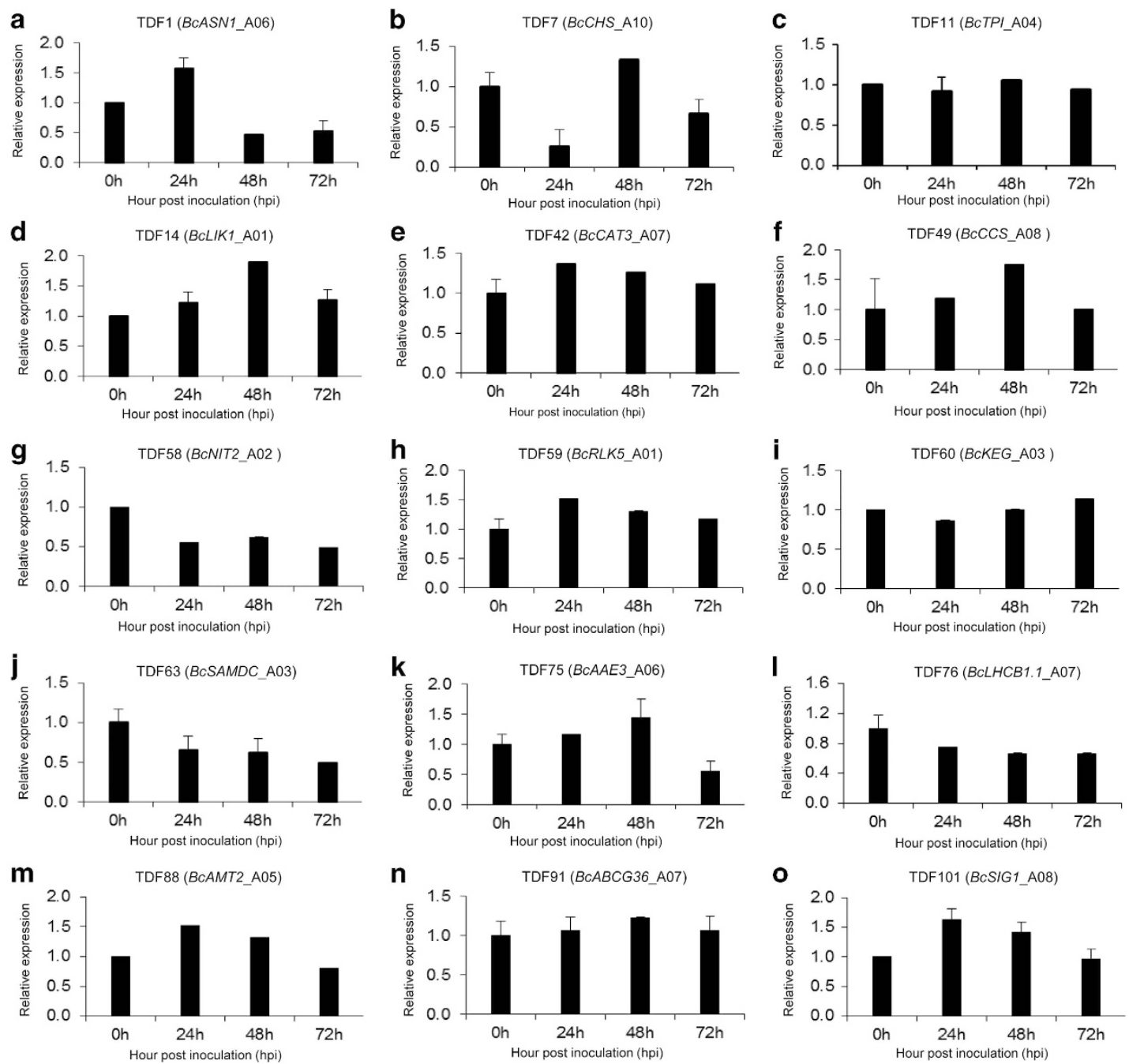

Figure 3. Quantitative real-time PCR analysis of 15 selected genes. (a-o) Both control and treated third leaf of five plants 'Suzhou Qing' were harvested and pooled at 0, 24, 48 and 72 h.p.i. The relative expression level for $H$. parasitica-inoculated plants at each time point was calculated as fold of the control plants at 0 using the LOG method. All data were normalized to the $\beta$-actin gene expression level. Error bars indicate s.d. of the three technical repeats. 
a

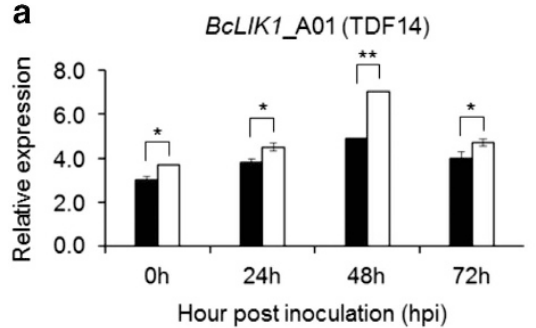

C

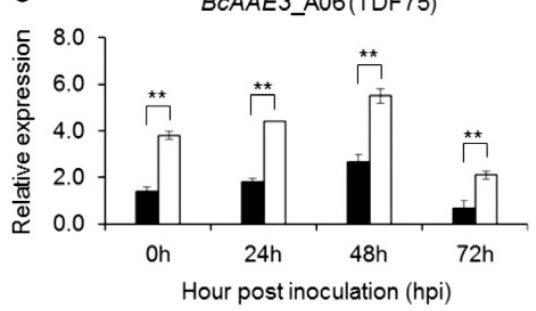

b

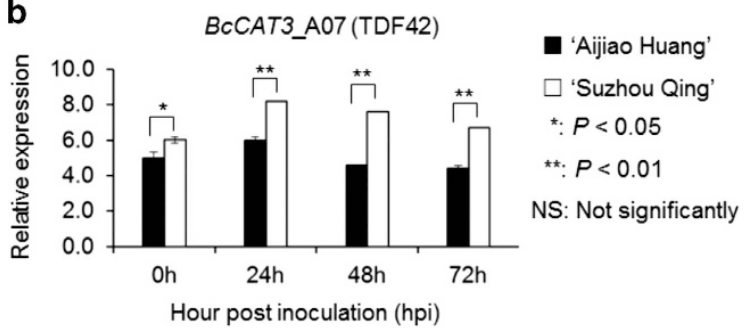

d

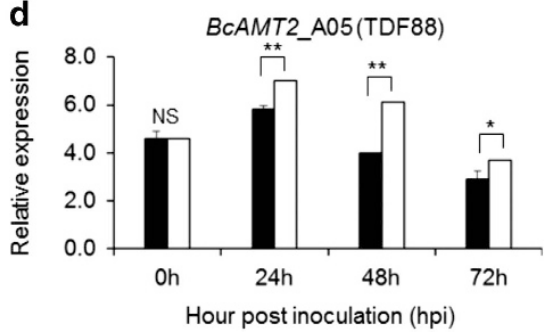

Figure 4. The comparison of partial TDFs (a-d) expression patterns between resistant line and susceptible line. The results show that these four genes has almost the same expression trend between two lines, but the expression of gene in the resistant line 'Suzhou Qing' is higher than that of in susceptible line 'Aijiao Huang'. Both control and treated third leaf of five plants were harvested and pooled at 0, 24,48 and $72 \mathrm{~h}$. p.i. The gene expression level was calculated using the LOG method. The gene expression level in both lines was compared using LOG value directly. All data were normalized to the $\beta$-actin gene expression level. Error bars indicate s.d. of the three technical repeats. Asterisks indicate statistically significant differences compared 'Aijiao Huang' and 'Suzhou Qing' at each TDFs (Student's $t$-test: ${ }^{*} P<0.05$; ${ }^{* *} P<0.01$ ).

expression of the genes related with fungal resistance in resistance line were higher than that in susceptible line.

\section{DISCUSSION}

We identified 129 TDFs, of which 121 TDFs were upregulated and eight were down-regulated using CDNA-AFLP. ${ }^{2,3}$ By BLAST searching in the Brassica database, these TDFs were classified according to their different functions. The functional categorization showed a complex linkage between proteins encoding by the TDFs. Information obtained from this study may provide a foundation for better understanding defense mechanisms of the non-heading Chinese cabbage with $H$. parasitica incompatible interaction.

\section{Defense}

Our data showed that several transcripts encoding the group of $P R$ proteins were differentially expressed in the interaction (Table 2). For example, TDF3 ( $\beta$-1, 3-glucanase) was induced within 24 h.p.i. and its expression peaked at 48 h.p.i. The expression levels of TDF8 (Hapless 8), TDF16 (a thaumatin-like protein) and TDF28 (PR 1-like protein) were induced within 24-72 h.p.i. Previously, we cloned the full length of $\beta$-1, 3-glucanase, hapless 8 and $P R 1$ genes, and analysed their expression patterns in response to $H$. parasitica infection in 'Suzhou Qing' cultivar of non-heading Chinese cabbage. ${ }^{10}$ The accumulations of these two transcripts were upregulated during the infection period, suggesting that these proteins may participate in the defence reaction for nonheading Chinese cabbage against $H$. parasitica. We also found that expression of TDF39 (ATMT-1) peaked at 48 h.p.i. In rice and barley, MT2A genes were induced by stresses such as drought, cold treatment and wounding or in response to pathogen attacks. ${ }^{12-14}$ Further research revealed that products of homologous MT scavenged the reactive oxygen species (ROS), such as $\mathrm{OH}$ to $\mathrm{H}_{2} \mathrm{O}$. ${ }^{15}$ Evidences suggest that the generation of ROS occurs at early stage in the plant-pathogen interaction. Rapid accumulation of ROS causes oxidative burst that results in hypersensitive cell death and cell wall cross-link. ${ }^{16}$ Our data may indicate that the upregulation of MT2A in non-heading Chinese cabbage leaves may contribute to ROS accumulation for inducing the hypersensitive response of the plant.

\section{Signal transduction}

Studies suggest that several signal transduction-related proteins are involved in the plant-fungus interactions..$^{1-3,17}$ We also identified many TDFs related to the signal transduction, such as TDF42 (Catalase 3), TDF43 (2-Cys PrxB), TDF45 (ATP binding), TDF50 (ATRER1A), TDF58 (Nitrilase), TDF48 (BCCAM3_A04, calcium ion binding) and TDF68 (WRKY DNA-binding protein).

Calcium binding-like proteins may have a role in signalling pathways against pathogens and wounding. ${ }^{18} \mathrm{~A}$ number of downstream targets of calmodulin $(C a M)$, including nitric oxide synthase, ${ }^{19}$ barley MLO protein, ${ }^{20}$ maize $\mathrm{Ca}^{2+}-\mathrm{CaM}^{21}$ and transcriptional regulators, ${ }^{22}$ are involved in plant responses to pathogens. Given that calcium ion-binding proteins are important modulators of defence response in pathways for pathogen sensing in plants, the CAM 3 gene could have a special role as $\mathrm{Ca}^{2+}$ sensors during the plant immune response to the fungus $H$. Parasitica.

WRKY proteins are signal transcriptional factors recognizing the TTGAC (C/T) W-box elements in the promoters of a large number of plant defence-related genes. ${ }^{23}$ Many of WRKY genes are upregulated particularly in pathogen-infected, wounded or abiotic-treated plants. ${ }^{24}$ In this study, expression of WRKY DNAbinding protein peaked at 24 h.p.i., suggesting that the possible role of WRKYs is in the regulation of the genes associated with plant defence responses. However, we found that expression pattern of TDF60 (WRKY gene) determined by qRT-PCR was inconsistent with that of cDNA-AFLP. The inconsistence may be caused by different paralogues in the genome.

\section{Regulation}

An ethylene response factor (BCERF1_A01, TDF93; Table 2), a regulator of ethylene responses after pathogen attack in Arabidopsis, may have a key role in the non-heading Chinese cabbage$H$. parasitica interaction. Previous studies have been demonstrated 
that ERFs are involved in regulating the expression of the defencerelated genes during the disease resistance responses. ${ }^{26,27}$

We found that TDF91 (BCABCG36_A07, ATP-binding cassette g36) was inhibited after inoculation by CDNA-AFLP analysis. However, its expression was induced weakly at 24 h.p.i., peaked at 48 h.p.i. and decreased weakly at 72 h.p.i. afterwards by qRT-PCR analysis. TDF100 (BCEDM1_A09) coding for an enhanced downy mildew 1 homolog was found to be induced during the infection period. Its relationship with the fungi, bacteria and viruses has been identified to be regulators of $R$ gene-mediated resistance in other crop species. ${ }^{28,29}$ Recent studies have revealed that EDM1 homologue gene SGT1 is required for pathogen-induced disease-associated cell death during both compatible and incompatible interactions in tobacco. ${ }^{30}$

\section{Energy metabolism}

Energy metabolism has an important role in plants-pathogen interaction. The photosynthetic carbon cycle (PCC) is part of the dark reactions of photosynthesis and can be roughly divided into three steps: carboxylation, reduction reaction and regeneration of $R u B P^{31}$ In this study, we found that some TDFs relating to energy metabolism were downregulated, such as TDF76 (chlorophyll $a / b$ binding protein), TDF78 (SIT4 phosphatase-associated family protein) and TDF82 (PP2C-related protein), whereas some were upregulated, such as TDF79 (receptor like protein 51), TDF80 (NADH-ubiquinone oxidoreductase), TDF83 (respiratory burst oxidase protein), TDF85 (rubisco small subunit 16 ; Table 2). Previous reports have identified that they are involved in PCC cycle, for example, TDF86 (reduction of transketolase) inhibited ribulose-1,5-bisphosphate regeneration and photosynthesis. ${ }^{31,32}$ These results are consistent with previous report that the expression of energy metabolism-related genes are induced and/or suppressed in photosynthesis during abiotic and biotic stresses. ${ }^{33,34}$ Our results may suggest that PCC cycle could provide protection function in energy metabolism during nonheading Chinese cabbage against $H$. parasitica.

\section{Protein-protein interaction}

A number of genes related to protein-protein interaction were induced after inoculation, such as TDF110 (BCTPR12_A07, tetratricopeptide repeat protein) and TDF111 (BCZF_A01, zinc-finger family protein). Of which, the gene expression of $P A T$ is induced in the presence of ozone in Arabidopsis. ${ }^{35}$ The tryptophan biosynthetic enzymes, including anthranilate synthase (ASA) and PAT, are co-ordinately upregulated at both the messenger RNA and protein level during biotic and abiotic stress. ${ }^{36}$ We found that one of $B C Z F$ orthologous to $A$. thaliana was induced after inoculation (Table 2). Rizhsky et al. speculate that a zinc-finger protein is required for the expression of ascorbate peroxidase, which provides some measure of resistance for plant during oxidative stress. ${ }^{37}$ In this study, fact that pathogen-induced accumulation of these proteinprotein interaction-related genes suggested that these genes may be involved in some defence mechanisms against $H$. parasitica indirectly.

Using the cDNA-AFLP method, we also detected several unknown functional genes. Their biological role is still unclear.

In this study, we examined gene expression patterns in an incompatible interaction between non-heading Chinese cabbage 'Suzhou Qing' and the downy mildew pathogen. We obtained 129 TDFs with different expression patterns and classified functional categories using CDNA-AFLP. Fifteen TDFs were randomly selected for validation of CDNA-AFLP expression patterns using qRT-PCR. Results showed that reliability of CDNA-AFLP is suitable for detecting differentially expressed genes. Among the 15 TDFs, four TDFs are related with fungal resistance, namely, TDF14 (BCLIK1_A01), TDF42 (BCCAT3_A07), TDF75 (BCAAE3_A06) and TDF88 (BCAMT2 A05). We further compared expression patterns in 'Suzhou Qing' and 'Aijiao Huang' using qRT-PCR. Results showed that the four genes displayed similar expression trend in the two lines. Importantly, the expression of genes in the resistant line is higher than that in susceptible line. These genes expression patterns and their putative functions may provide insight in understanding the non-heading Chinese cabbage-downy mildew incompatible interaction. Our study may also provide a foundation for better understanding molecular mechanisms and can be beneficial in selecting candidate resistance genes for the incompatible interaction between non-heading Chinese cabbage and $H$. Parasitica. Further research is needed to study the comparison between compatible and incompatible interactions to identify novel and common genes that regulate non-heading Chinese cabbage-downy mildew pathosystem.

\section{CONFLICT OF INTEREST}

The authors declare no conflict of interest.

\section{ACKNOWLEDGEMENTS}

The research was supported by the following: the Independent Innovation Fund for Agricultural Science and Technology of Jiangsu Province (CX (15)1015); the Sciencetechnology Support Plan of Jiangsu Province (BE2012325, BE2013429); Natural Science Foundation of China (31272173); and Jiangsu Natural Science Foundation (BK20140704).

\section{REFERENCES}

1 Takemoto D, Hardham AR. The cytoskeleton as a regulator and target of biotic interactions in plants. Plant Physiol 2004; 136: 3864-3876.

2 Pitino M, Armstrong CM, Duan YP. Rapid screening for citrus canker resistance employing pathogen-associated molecular pattern-triggered immunity responses. Hortic Res 2015; 2: 15042.

3 Amrine $\mathrm{KCH}$, Blanco-Ulate $\mathrm{B}$, Riaz S, Pap D, Jones L, Figueroa-Balderas $\mathrm{R}$ et al. Comparative transcriptomics of Central Asian Vitis vinifera accessions reveals distinct defense strategies against powdery mildew. Hortic Res 2015; 2: 15037.

4 Monot C, Pajot E, Le Corre D, Silué D. Induction of systemic resistance in broccoli (Brassica oleracea var. botrytis) against downy mildew (Peronospora parasitica) by avirulent isolates. Biol Control 2002; 24: 75-81.

5 Farinhó M, Coelho P, Carlier J, Svetleva D, Monteiro A, Leitão J. Mapping of a locus for adult plant resistance to downy mildew in broccoli (Brassica oleraceacon var. italica). Theor Appl Genet 2004; 109: 1392-1398.

6 Casimiro S, Tenreiro R, Monteiro AA. Identification of pathogenesis-related ESTs in the crucifer downy mildew oomycete Hyaloperonospora parasitica by highthroughput differential display analysis of distinct phenotypic interactions with Brassica oleracea. J Microbiol Methods 2006; 66: 466-478.

7 Tang YQ, Yu SC, Zhu YL, Zhang FL, Yu YJ, Zhao XY et al. Construction and analysis of suppression subtractive hybridization CDNA library in Chinese cabbage (Brassica rapa ssp. pekinensis) leaves induced by Peronospora parasitica. Plant Physiol Commun 2010; 46: 453-458.

8 De Paepe A, Vuylsteke M, Van Hummelen P, Zabeau M, Van Der Straeten D. Transcriptional profiling by CDNA-AFLP and microarray analysis reveals novel insights into the early response to ethylene in Arabidopsis. Plant $J$ 2004; 39: 537-559.

9 Sarosh BR, Meijer J. Transcriptional profiling by cDNA-AFLP reveals novel insights during methyl jasmonate, wounding and insect attack in Brassica napus. Plant Mol Biol 2007; 64: 425-438.

10 Chen X, Hou X, Zhang J, Zheng J. Molecular characterization of two important antifungal proteins isolated by downy mildew infection in non-heading Chinese cabbage. Mol Biol Rep 2008; 35: 621-629.

11 Livak KJ, Schmittgen TD. Analysis of relative gene expression data using real-time quantitative PCR and the $2^{-\triangle \Delta C T}$ method. Methods 2001; 25: 402-408.

12 Ozturk ZN, Talamé V, Deyholos M, Michalowski CB, Galbraith DW, Gozukirmizi N et al. Monitoring large-scale changes in transcript abundance in drought- and salt-stressed barley. Plant Mol Biol 2002; 48: 551-573.

13 Jin S, Cheng Y, Guan Q, Liu D, Takano T, Liu S. A metallothionein-like protein of rice (rgMT) functions in $E$. coli and its gene expression is induced by abiotic stresses. Biotechnol Lett 2006; 28: 1749-1753.

14 Degenhardt J, Al-Masri AN, Kürkcüoglu S, Szankowski I, Gau AE. Characterization by suppression subtractive hybridization of transcripts that are differentially expressed in leaves of apple scab-resistant and susceptible cultivars of Malus domestica. Mol Genet Genomics 2005; 273: 326-335. 
15 Akashi K, Nishimura N, Ishida Y, Yokota A. Potent hydroxyl radical-scavenging activity of drought-induced type-2 metallothionein in wild watermelon. Biochem Biophys Res Commun 2004; 323: 72-78.

16 Das R, Roy A, Dutta N, Majumder H. Reactive oxygen species and imbalance of calcium homeostasis contributes to curcumin induced programmed cell death in Leishmania donovani. Apoptosis 2008; 13: 867-882.

17 Rushton PJ, Torres JT, Parniske M, Wernert P, Hahlbrock K, Somssich IE. Interaction of elicitor-induced DNA-binding proteins with elicitor response elements in the promoters of parsley PR1 genes. EMBO J 1996; 15: 5690-5700.

18 Cheong YH, Chang HS, Gupta R, Wang X, Zhu T, Luan S et al. Transcriptional profiling reveals novel interactions between wounding, pathogen, abiotic stress, and hormonal responses in Arabidopsis. Plant Physiol 2002; 129: 661-677.

19 Zeidler D, Zähringer U, Gerber I, Dubery I, Hartung T, Bors W et al. Innate immunity in Arabidopsis thaliana: Lipopolysaccharides activate nitric oxide synthase (NOS) and induce defense genes. Proc Natl Acad Sci USA 2004; 101: 15811-15816.

20 Kim MC, Panstruga R, Elliott C, Muller J, Devoto A, Yoon HW et al. Calmodulin interacts with MLO protein to regulate defence against mildew in barley. Nature 2002; 416: 447-451.

21 Hu X, Jiang M, Zhang J, Zhang A, Lin F, Tan M. Calcium-calmodulin is required for abscisic acid-induced antioxidant defense and functions both upstream and downstream of $\mathrm{H}_{2} \mathrm{O}_{2}$ production in leaves of maize (Zea mays) plants. New Phytol 2007; 173: 27-38.

22 Yang T, Poovaiah BW. Hydrogen peroxide homeostasis: activation of plant catalase by calcium/calmodulin. Proc Natl Acad Sci USA 2002; 99: 4097-4102.

23 Turck F, Zhou A, Somssich IE. Stimulus-dependent, promoter-specific binding of transcription factor WRKY1 to its native promoter and the defense-related gene PCPR1-1 in Parsley. Plant Cell 2004; 16: 2573-2585.

24 Pandey SP, Somssich IE. The role of WRKY transcription factors in plant immunity. Plant Physiol 2009; 150: 1648-1655.

25 Berrocal-Lobo M, Molina A, Solano R. Constitutive expression of ETHYLENERESPONSE-FACTOR1 in Arabidopsis confers resistance to several necrotrophic fungi. Plant J 2002; 29: 23-32.

26 Singh KB, Foley RC, Oñate-Sánchez L. Transcription factors in plant defense and stress responses. Curr Opin Plant Biol 2002; 5: 430-436.

27 Cao $Y$, Wu Y, Zheng Z, Song F. Overexpression of the rice EREBP-like gene OSBIERF3 enhances disease resistance and salt tolerance in transgenic tobacco. Physiol Mol Plant Pathol 2005; 67: 202-211.
28 Lingelbach LB, Kaplan KB. The interaction between Sgt1p and Skp1p is regulated by HSP90 chaperones and is required for proper CBF3 assembly. $\mathrm{Mol} \mathrm{Cell} \mathrm{Biol}$ 2004; 24: 8938-8950.

29 Holt BF, Belkhadir Y, Dangl JL. Antagonistic dontrol of disease resistance protein stability in the plant immune system. Science 2005; 309: 929-932.

30 Wang K, Uppalapati SR, Zhu X, Dinesh-Kumar SP, Mysore KS. SGT1 positively regulates the process of plant cell death during both compatible and incompatible plant-pathogen interactions. Mol Plant Pathol 2010; 11: 597-611.

31 Yokota A, Shigeoka S, Hans J, Bohnert HN, Norman GL. Engineering photosynthetic pathways. Adv Plant Biochem Mol Biol 2008; 1: 81-105.

32 Henkes S, Sonnewald U, Badur R, Flachmann R, Stitt M. A small decrease of plastid transketolase activity in antisense tobacco transformants has dramatic effects on photosynthesis and phenylpropanoid metabolism. Plant Cell 2001; 13: 535-551.

33 Seki M, Narusaka M, Ishida J, Nanjo T, Fujita M, Oono $Y$ et al. Monitoring the expression profiles of 7000 Arabidopsis genes under drought, cold and highsalinity stresses using a full-length cDNA microarray. Plant J 2002; 31: 279-292.

34 Scharte J, SchÖN H, Weis E. Photosynthesis and carbohydrate metabolism in tobacco leaves during an incompatible interaction with Phytophthora nicotianae. Plant Cell Environ 2005; 28: 1421-1435.

35 Conklin PL, Last RL. Differential accumulation of antioxidant mRNAs in Arabidopsis thaliana exposed to ozone. Plant Physiol 1995; 109: 203-212.

36 Zhao J, Last RL. Coordinate regulation of the tryptophan biosynthetic pathway and indolic phytoalexin accumulation in Arabidopsis. Plant Cell 1996; 8: 2235-2244.

37 Mittler R, Vanderauwera S, Gollery M, Van Breusegem F. Reactive oxygen gene network of plants. Trends Plant Sci 2004; 9: 490-498.

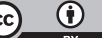

This work is licensed under a Creative Commons Attribution 4.0 International License. The images or other third party material in this article are included in the article's Creative Commons license, unless indicated otherwise in the credit line; if the material is not included under the Creative Commons license, users will need to obtain permission from the license holder to reproduce the material. To view a copy of this license, visit http://creativecommons.org/licenses/ by/4.0/

(c) The Author(s) 2016

.

